\title{
Experimental Evaluation of Antenna Polarization and Elevation Effects on Drone Communications
}

\author{
Mahmoud Badi, John Wensowitch, Dinesh Rajan, and Joseph Camp \\ Southern Methodist University \\ \{mbadi,jwensowitch,rajand,camp\}@smu.edu
}

\begin{abstract}
In the next wave of swarm-based applications, unmanned aerial vehicles (UAVs) need to communicate with peer drones in any direction of a three-dimensional (3D) space. On a given drone and across drones, various antenna positions and orientations are possible. We know that, in free space, high levels of signal loss are expected if the transmitting and receiving antennas are cross polarized. However, increasing the reflective and scattering objects in the channel between a transmitter and receiver can cause the received polarization to become completely independent from the transmitted polarization, making the cross-polarization of antennas insignificant. Usually, these effects are studied in the context of cellular and terrestrial networks and have not been analyzed when those objects are the actual bodies of the communicating drones that can take different relative directions or move at various elevations. In this work, we show that the body of the drone can affect the received power across various antenna orientations and positions and act as a local scatterer that increases channel depolarization, reducing the cross-polarization discrimination (XPD). To investigate these effects, we perform experimentation that is staged in terms of complexity from a controlled environment of an anechoic chamber with and without drone bodies to in-field environments where drone-mounted antennas are in-flight with various orientations and relative positions with the following outcomes: (i.) drone relative direction can significantly impact the XPD values, (ii.) elevation angle is a critical factor in 3D link performance, (iii.) antenna spacing requirements are altered for co-located cross-polarized antennas, and (iv.) cross-polarized antenna setups more than double spectral efficiency. Our results can serve as a guide for accurately simulating and modeling UAV networks and drone swarms.
\end{abstract}

\section{INTRODUCTION}

The commercial use of UAVs, in addition to military applications, has been exponentially increasing. In 2017, the number of commercial drones increased by $58 \%$ in North America compared to 2016 with a projected global market of $\$ 11.61$ billion by 2022 [1]. With the new advancements in UAVs such as the ability to hover and rapidly change locations and respond to control commands, UAVs are becoming more attractive to many organizations in a diversified

Permission to make digital or hard copies of all or part of this work for personal or classroom use is granted without fee provided that copies are not made or distributed for profit or commercial advantage and that copies bear this notice and the full citation on the first page. Copyrights for components of this work owned by others than ACM must be honored. Abstracting with credit is permitted. To copy otherwise, or republish, to post on servers or to redistribute to lists, requires prior specific permission and/or a fee. Request permissions from permissions@acm.org.

MSWiM '19, November 25-29, 2019, Miami Beach, FL, USA

(c) 2019 Association for Computing Machinery.

ACM ISBN 978-1-4503-6904-6/19/11 . \$ \$15.00

https://doi.org/10.1145/3345768.3355916 array of applications (e.g., inspection, mapping, monitoring). The next wave of these and other applications will be coordination of drone swarms to achieve automated tasks, leading researchers to extensively study UAV-based communications. The majority of literature, however, focuses on simulations ([2] and references within) or optimization models that target the optimal placement of a UAV [3-6] in various applications. These methods are important research tools and give valuable insight but may be misleading if these tools are not grounded in reality. Recently, measurement-based studies have been conducted with the majority of them focusing on air-toground (AtG) communications [7-13]. Experiments that investigate air-to-air (AtA) links mainly focus on received signal variations with distance and finding path-loss exponents with little emphasis on the body effects, antenna radiation pattern, polarization, and their joint effects. For example, authors in [14] measure the received signal strength (RSS) over various altitudes and empirically model the ground effects on multipath propagation. Furthermore, authors in [15] investigate network performance of AtA and AtG links in different network topologies and measure throughput at various distances and find the path-loss exponent. More elaborate details on related work are presented in $\S 5$.

As drones are expected to communicate in swarms, carry $5 \mathrm{G}$ traffic, and be integrated in IoT applications [6, 9], drone-based MIMO systems that offer higher throughput and more robust airborne links are becoming more attractive than ever [11-13]. However, it is well known that the capacity of these MIMO systems can be significantly reduced due to high spatial correlation of its channels $[26,33]$. The MIMO spatial correlation is known to largely depend on antenna spacing, radiation pattern and polarization; factors that are found to be crucial when modeling polarized MIMO [25-28] channels in 3D. These factors are of particular interest to us because of the unique form factor and the potential effects of the body on the radiation pattern and polarization, the ability to move freely in 3D space (i.e., in elevation and azimuth planes), and the limited space on UAVs, which might pose a challenge on antenna spacing and correlation in MIMO applications.

Cross-polarization discrimination (XPD) has a significant impact on the spatial correlation of the MIMO channel [26, 33] and according to the geometrical theory of channel depolarization [24] and measurement campaigns [25], depends largely on the environment and local scatterers around a receiving body. However, none of the current works that model 3D UAV channels [30, 31] investigate and quantify the impact of the drone body on XPD. In this paper, we experimentally measure how the drone body can affect the received power and XPD in different blocking scenarios. Furthermore, as elevation between drones can cause polarization mismatch and consequent power losses, we measure the RSS in a UAV-to-UAV link at different elevation angles for six different 
antenna orientation combinations to determine which antenna orientations are best to cover movement in 3D space. Based on our results, we accurately model drone networks with an awareness of antenna spacing and orientations and relative elevations for use in simulation or real-world deployments. Our contributions in this paper are as follows.

- We build a UAV-based $1 \times 2$ diversity system using Software Defined Radio (SDR) to explore the effects of the UAV body, elevation and antenna orientation on the performance of UAV-to-UAV links.

- In an anechoic chamber, we experimentally characterize the effects of the designed UAV platform and show that the drone body significantly impacts channel depolarization and reduce XPD by an average of $14.5 \mathrm{~dB}$ over all azimuth directions compared to an isolated antenna scenario.

- We perform in-field measurements and characterize the body-induced losses on RSS when two drones move in different azimuth directions at the same altitude. We show that the relative direction of one drone to another can reduce RSS by up to $16 \mathrm{~dB}$ compared to when the two drones are exactly facing each other. Based on this finding, we propose a range of body excess loss that should be added to the conventional log-distance path-loss model for more accurate predictions of UAV-to-UAV links. This model is shown to achieve a lower absolute error in prediction by $89 \%$ compared to the conventional model.

- We perform in-field measurements on the impact of the elevation angle on RSS and SNR improvements due to diversity for six different antenna orientation combinations. We find that when the two drones are facing each other, performance of UAV-to-UAV links in 3D space is mainly driven by the elevation angle. We show that the average RSS can change by more than $30 \mathrm{~dB}$, even when the polarization is matched.

- We provide recommendations on antenna placement and orientations based on our measurement results. We find that, in general, a $\frac{2}{3} \lambda$ is enough spacing to achieve a correlation coefficient that is less than 0.7. Furthermore, in order to achieve the best performance in UAV-to-UAV links in 3D space, we show that horizontal antennas perform better than vertical antennas when near locations that are above or below the transmit drone while other locations are best covered by vertical antennas.

This paper is organized as follows. We present a baseline understanding of the effects of the drone body on co-polarized and crosspolarized channels and XPD degradation in $\S 2$. In $\S 3$, we report results from in-field experiments that demonstrate the impact of the drone body and relative direction on RSS and cross-polarized channels and propose an initial model that takes body-induced losses into account to provide more accurate large-scale fading predictions. Then, in $\S 4$, we discuss correlation, antenna placement, and the impact of elevation angle on RSS and diversity. Related work is discussed in $\S 5$, and we conclude in $\S 6$.

\section{BASELINE UNDERSTANDING OF DRONE BODY EFFECTS USING ANECHOIC CHAMBER MEASUREMENTS}

To begin our experimentation, we first characterize the body-induced effects of the drone on the radiation pattern in the elevation and azimuth planes for co-polarized (co-pol) and cross-polarized (x-pol) channels. Understanding the body-induced effects on radiation pattern and XPD can be crucial for researchers in establishing and analyzing models for polarized MIMO channels [25-31] that take radiation pattern, XPD, and correlation coefficients into consideration. These factors can affect drone-based design decisions such as antenna placement, orientation, and optimal location of a drone in 3D space. Therefore, in this section, we aim to address these issues. The experiments are carried out in an anechoic chamber, and the results are presented for two main scenarios: ( $i$ ) Isolated, and (ii) Drone-mounted. In the isolated scenario, the radiation pattern of the dipole antenna is characterized with the antenna being mounted without the drone. In the drone-mounted scenario, the antenna is mounted on the drone and the same procedure for determining the radiation pattern is repeated. For a fair comparison between the two scenarios, the same dipole antenna is used and the same measurement rules [23] are followed with the drone configuration matching that of the in-field experimentation in the following section. The setup for the drone-based scenario is shown in Fig. 2.

\subsection{Body Effects on Co-Polarized Channels}

Since polarization mixing between the horizontal and vertical components of an electromagnetic wave can occur because of antenna imperfections or due to the propagation environment (channel depolarization) [24, 25], it is useful to show the radiation pattern of the two scenarios (isolated and drone mounted) so that the channelinduced effects, which are caused by the body of the drone, can be quantified. We start by analyzing the drone-body effects on the co-polarized channel, and then move to the cross-polarized channel and present the measured XPD, analyzing how it can be significantly reduced by the drone body.

Here, we study the effects of the drone body on the co-polarized azimuth and elevation radiation patterns. The azimuth radiation pattern is obtained by rotating the platform over the $\phi$ direction (azimuth rotation) and capturing the received power in the $P_{V V}$ channel. The elevation radiation pattern is obtained by the same procedure for the $P_{H H}$ channel; VV indicates that the transmitting and receiving antennas are both vertically polarized, and $\mathrm{HH}$ means they are both horizontally polarized. Note that it is widely accepted in literature [24, 26, 27] (and verified by our measurements, but omitted due to limited space) that $P_{V V}=P_{H H}$ and $P_{V H}=P_{H V}$. This reduces the complexity in getting the elevation pattern when the antenna is mounted on the drone. The automatic rotation in the chamber is in $1.8^{\circ}$ increments from $0^{\circ}$ to $360^{\circ}$ and the received power level is captured for every $1.8^{\circ}$. The received power levels are then normalized to the maximum power level and the results are plotted. The radiation patterns for the two scenarios (isolated and drone-mounted) are compared against each other in the VV and $\mathrm{HH}$ channels. Figs. 1(a) and 1(b) show the results.

It can be clearly seen that both the elevation and azimuth patterns are affected by the drone body, at some angles more significantly 


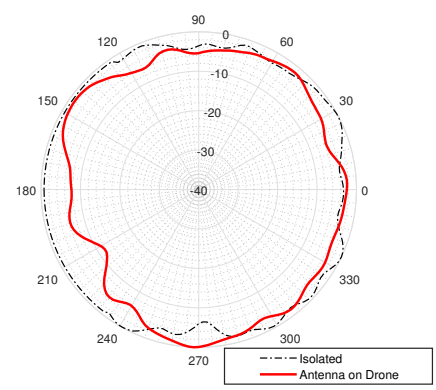

(a)

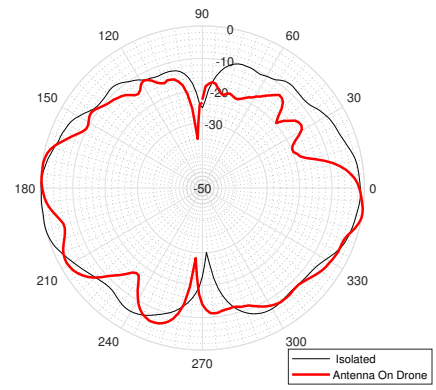

(b)

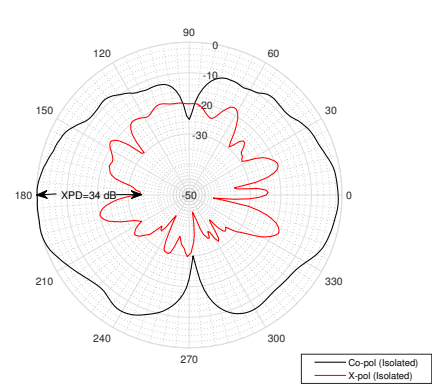

(c)

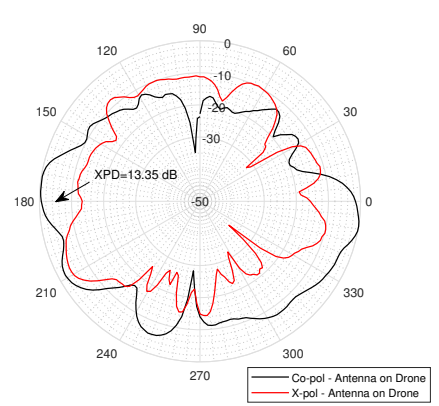

(d)

Figure 1: Effects of the drone body on co-polarized azimuth (a) and elevation (b) radiation patterns for two scenarios: isolated (no drone), and drone-mounted. Effects of the drone body on cross-polarized power levels and XPD when the receiving antenna is isolated (c) and drone-mounted (d).

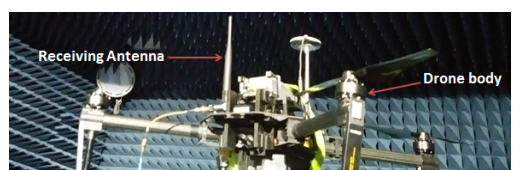

Figure 2: Anechoic chamber characterization of the radiation pattern when the antenna is mounted on the drone (Drone-mounted scenario) for the $P_{V V}$ channel.

than others. In the azimuth plane, we can see that the drone body can reduce the received power by a maximum of $11 \mathrm{~dB}$ approximately when the drone is facing away (around $\phi=210^{\circ}$ ). This result agrees with many other findings in literature [9-11, 14]. In the elevation plane, we can see that the effect of the body is more significant and less symmetrical when compared to the isolated case. We can see that the received power when the antenna is mounted on the drone can be reduced by up to (max difference) $18 \mathrm{~dB}$ compared to the isolated case. It is worth mentioning that the same experiments were conducted for another antenna position on the drone (right position in Fig. 2) and, albeit a different radiation pattern was obtained, similar differences in values (average and standard deviations) were found.

The min, max, average, and standard deviation of the difference in power levels between the isolated and the drone-mounted case in the azimuth and elevation planes are denoted by $\mathrm{E}_{\text {azimuth }}$ and $\mathrm{E}_{\text {elevation }}$ and defined as $P_{V V \text {,isolated }}-P_{V V \text {,drone-mounted }}$ and $P_{H H, \text { isolated }}-P_{H H, d r o n e-m o u n t e d}$ for the azimuth and elevation planes, respectively. The results are summarized in Table 1.

Table 1: Body-Induced Losses on co-polarized azimuth and elevation power levels

\begin{tabular}{|c|c|c|c|c|}
\hline Plane & Min. & Max. & Avg. & SD \\
\hline$£_{\text {azimuth }}(d B)$ & 0.016 & 10.96 & 3.03 & 2.53 \\
\hline$£_{\text {elevation }}(d B)$ & 0.02 & 18.72 & 4.13 & 4.06 \\
\hline
\end{tabular}

\subsection{Body Effects on Cross-Polarized Channels}

In this section, we analyze the impact of the drone body on the cross-polarization discrimination (XPD), which describes how well the two orthogonal polarization components can be separated. XPD is calculated as the ratio of the amount of power received in the co-polarized versus cross-polarized directions [28]:

$$
X P D=P_{\text {copol }} / P_{x p o l}
$$

Here, we present results of the $P_{H H}$ (black solid lines in Figs. 1(c) and $1(\mathrm{~d})$ ) and $P_{V H}$ (red lines in the same figures) and calculate the difference between the two as the angle-specific XPD value. It is well known that channel induced XPD can vary depending on the distance, environment (LOS or NLOS), and the angle between transmitters and receivers[25]. This is because of the variations in the number and nature of scatterers along the path between the transmit and receive antennas. In scenarios where multipath does not exist, and for identical antennas at the same orientation angle, the XPD is found to be approximately constant and independent of distance [32]. Therefore, it is expected that a higher amount of polarization mixing might occur due to reflections from the drone body surrounding the antenna, and, as a result, lower XPD values are expected in the drone-mounted scenario. From Fig. 1(d) we can see a clear impact of the drone body on the cross-polarized power measured by the receiving antenna when it is mounted on the drone. Even when the receiving antenna (when drone-mounted) is directly facing the transmitter $\left(\phi=0^{\circ}\right)$, the drone body can reduce the the XPD by approx. $9 \mathrm{~dB}$ (from $24 \mathrm{~dB}$ in the isolated scenario to $15 \mathrm{~dB}$ when drone-mounted). More significant reduction appears when the drone is facing-away with an XPD reduction by 20.6 $\mathrm{dB}$. We find that, on average over all angles, the XPD is reduced from $19.2 \mathrm{~dB}$ in the isolated case to $4.6 \mathrm{~dB}$ when drone-mounted. In addition, the maximum XPD in the isolated case is found to be $41.15 \mathrm{~dB}$ as opposed to $28.02 \mathrm{~dB}$ in the drone-mounted case. Results are summarized in Table 2. These are significant findings due to the impact that XPD can have on correlation and achieved capacity in MIMO [33] or diversity applications [34] that leverage differently-polarized channels. For example, an average XPD value of $0 \mathrm{~dB}$ means that the rank of the MIMO channel is 1 . Hence, spatial multiplexing is not possible [33]. On the other hand, the same $0 \mathrm{~dB}$ value can indicate a richness of scatterers in the multipath environment, which leads to a low correlation coefficient and high diversity gains [29]. Surprisingly, and to the best of our knowledge, there is no prior work that characterizes polarization mixing and XPD degradation due to the sole effects of the drone body.

Table 2: XPD in The Isolated and Drone-mounted Scenarios

\begin{tabular}{|c|c|c|c|c|}
\hline Setup & Min. & Max. & Avg. & SD \\
\hline$X P D_{\text {isolated }}(d B)$ & -10.14 & 41.15 & 19.22 & 8.17 \\
\hline$X P D_{\text {drone-mounted }}(d B)$ & -19.23 & 28.02 & 4.61 & 8.41 \\
\hline
\end{tabular}




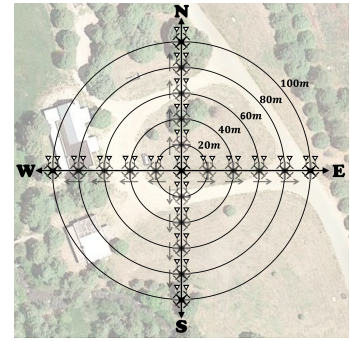

(a) Location(50-110 m height).

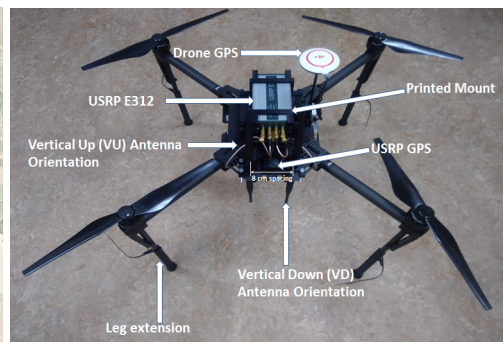

(b) Receiver drone setup.
Figure 3: In-field experiment location with receiver in center and transmitter in pictured locations along four cardinal directions (a). UAV-based SDR platform for the receiver drone with two (VU and VD) of the three antenna orientations(b).

After understanding the effect of the drone body on the received power in polarized channels, it is now possible to experimentally analyze (in-field) UAV-to-UAV links with different antenna orientations and at different elevation angles to understand the impact of the drone body and its location in space on performance.

\section{IN-FIELD EXPERIMENTS: BODY-INDUCED EFFECTS ON POLARIZED AIR-TO-AIR CHANNELS}

In this section, we investigate the body-induced effects on the received signal strength when the transmitting drone moves in four relative directions (North, South, East, and West) to the receiving drone; this means that it can be in front of (North), on the side (East or West), or behind (South) the receiving drone. Thus, the RSS may be affected differently depending on the transmitter's relative location. First, we explain the experimental setup and procedure. Then, we discuss measurement results and show how a prediction that is based only on a log-distance path loss model can significantly differ from the measured values because of the drone body-induced effects. Finally, we quantify these effects and give a range of values of excess loss that might be added to the conventional path-loss model to obtain more accurate predictions of the RSS in UAV-toUAV links. The results presented here can be integrated in simulated or in-field environments where multiple different connections (e.g., in swarms) are needed on different sides of the drone and relative direction becomes an important aspect in decisions made by drones that aim to optimize their links [19].

\subsection{Hardware and Software Setup}

We use the Universal Software Radio Peripheral (USRP) Ettus E312, which is a battery-operated $2 \times 2$ MIMO software defined radio platform with an operational frequency range of $70 \mathrm{MHz}-6 \mathrm{GHz}$ and up to $56 \mathrm{MHz}$ of instantaneous bandwidth. The transmission power of the USRP at $2.5 \mathrm{GHz}$ was calibrated by connecting the USRP to a Rohde and Schwarz FSH8 Spectrum Analyzer using an SMA connector and a $50 \Omega$ cable, and a measured value of $6.2 \mathrm{dBm}$ was recorded. Then, using GNU Radio blocks and through a Python script, we configure the transmitter to send a constant envelope sinusoid at a sampling rate of $32 \mathrm{k}$ samples/second and build a receiver that can simultaneously record the received signal on two receiving $\mathrm{RF}$ chains through two antennas mounted at a distance of $\frac{2}{3} \lambda$ where $\lambda$ is the wavelength of the transmitted carrier. We develop shell scripts that perform GPS logging and capture velocity, altitude, and IMU data while the drones are hovering. These sensor measurements are then used in splicing the data sets for analysis.

Using a ROBO 3D printer and MatterControl (printing software), we design and 3D print mounts for the USRP and antennas to be installed on a DJI Matrice 100 drone. Three different antenna mounts are built: vertical up (VU), horizontal $(\mathrm{H})$, and vertical down (VD). In this set of experiments, the VU and $\mathrm{H}$ orientations are implemented at the receiver while the transmitting drone has a VU antenna orientation. The VD mount is used for the next set of experiments where we explore elevation effects. These antenna mounts are securely fastened to the central frame on the front of the Matrice 100. The placement decision was based on the measured stability against vibrations while maintaining the load of the USRP at the center of the drone body. Furthermore, it is often desired to focus electromagnetic energy at some directions more than others, which might dictate the placement decisions of the antennas on a drone $[9,10,12]$ to be on a certain side. The in-phase $\left(r_{I}\right)$ and quadrature $\left(r_{Q}\right)$ components of the received signal of both RF chains are stored in a .dat file. Then, the signal envelope $|r|$ is obtained as $|r|=\sqrt{r_{I}^{2}+r_{Q}^{2}}$. The received power is calculated and a per-location analysis is carried out according to sensor measurements.

\subsection{Experiment Procedure}

Throughout this set of experiments, the heading direction (which corresponds to the locations of the antennas in our experiments) for both UAVs is North. We fly the receiving UAV and let it continuously hover at a $60-\mathrm{m}$ altitude in the same location (fixed) throughout all experiments while the transmitting UAV moves in the same plane. The transmitting drone starts at $20 \mathrm{~m}$ away in each cardinal direction (North, South, East, and West) and then flies in $20-\mathrm{m}$ increments away from the receiving drone until it reaches $100 \mathrm{~m}$ of separation distance, creating 5 distinct hovering locations per direction, each at a height of $60 \mathrm{~m}$ (i.e., these hovering locations are all at the same altitude). We define each experiment by its corresponding relative transmit drone direction. For example, an East experiment means that, with both UAVs facing North, the transmitting UAV moves linearly, $20 \mathrm{~m}$ away from the receiving drone in the East direction (see Fig. 3(a)).

\subsection{Relative Direction and Body Effects on RSS}

Given that no obstacles exist in the path between the two UAVs with negligible ground effects on multipath at $60 \mathrm{~m}$ altitude [14], one would expect that the relative direction of one drone to the other would not result in significant variations in received signal levels. Interestingly, our results show that the relative direction of the drone and its body placement with respect to its receiving antennas can result in a different RSS of up to $16 \mathrm{~dB}$. If we look at the results of the least squares fitting of the average RSS values in Fig. 4, we see how signal reception can significantly vary according to the relative direction of the transmitting drone. For example, at $40 \mathrm{~m}$ distance, when the transmit drone is in the relative North direction, the average received signal is $12 \mathrm{~dB}$ higher than when it is in the South direction. This additional loss due to the drone body 
Figure 4: Comparing the measured average RSS to the predicted values using the conventional PL model

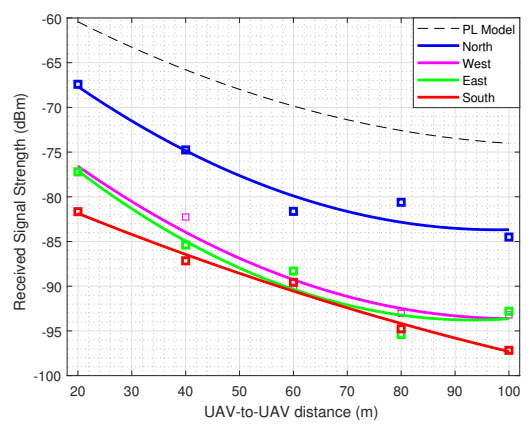

orientation agrees well with our expectations from the anechoic chamber measurements in Fig. 1(a), which indicates that a $10 \mathrm{~dB}$ loss in received power is expected when facing away $\left(\phi=180^{\circ}\right)$ from the transmitter. A slight difference from anechoic measurements is reasonable and expected because of flight vibrations and real environment conditions. Furthermore, we analyze this reduction in RSS on estimated throughput and find that, with an average noise floor level of $-110 \mathrm{dBm}$ measured at the same altitude and location, the average estimated spectral efficiency can be reduced from 11.62 bps $/ \mathrm{Hz}$ to $7.64 \mathrm{bps} / \mathrm{Hz}$, a reduction that can be significant when throughput is a concern in drone swarms.

Furthermore, the East and West directions are found to result in approximately the same average values of the RSS at all hovering locations. The range of these average values lies below the North direction and slightly above the South direction, implying less obstruction compared to South but more compared to North. The average of the RSS standard deviations over all hovering location for the North, South, East, and West are $3.32 \mathrm{~dB}, 4.29 \mathrm{~dB}, 2.29 \mathrm{~dB}$, and $1.86 \mathrm{~dB}$, respectively. From these results, we conclude that when two drones have their transmit/receive antennas mounted on the same side and facing the same direction, the relative direction of the transmitter to the receiver, and, consequently, the receiver's body placement with respect to its antennas can result in two distinct cases where the average RSS can change by up to $15 \mathrm{~dB}$. This finding can be crucial when drones autonomously attempt to optimize their spatial location in terms of estimated channel parameters [19].

\subsection{UAV Body Effects on Cross-Polarized Channels}

Interestingly, the relative direction of one drone to another is found to affect also the cross-polarized components. Here, we investigate the extent to which relative direction and body blockage can affect channel depolarization in an in-flight scenario as opposed to in an anechoic chamber (Fig. 1(d)), which showed that at $0^{\circ}$ elevation, the horizontally-oriented antenna (same antenna at exactly the same position) is expected to receive $13 \mathrm{~dB}$ to $15 \mathrm{~dB}$ less power compared to the vertically-oriented antenna. However, even though the two antennas ( $\mathrm{V}$ and $\mathrm{H}$ ) are identical, the spacing between them makes it difficult to distinguish the depolarization due to radiation pattern differences from the depolarization that occurred due to actual channel scatterers. Therefore, we do not claim that the following analysis provide exact XPD values but rather show an interesting trend where the difference in received power between the co-polarized and cross-polarized links can greatly differ based on the relative direction of the transmitter and the resulting body blockage at the receiver. We follow [32] in determining the polarization decoupling between the two orthogonal components as follows. We calculate the path loss for the two links as $P L_{V V}(d)(d B)=P_{t, v}-P_{r, v}$ and $P L_{V H}(d)(d B)=P_{t, v}-P_{r, h}$, where $d$ is the distance between the two drones for each of the four relative directions of the transmit UAV, $P_{t, v}$ is the transmit power (in $\mathrm{dBm}$ ) of the vertically-oriented antenna at the transmit drone, $P_{r, v}$ and $P_{r, h}$ are the received power levels of the vertical and horizontal receiving antennas, respectively. Now, the difference between the cross-polarized $(\mathrm{H})$ and co-polarized $(\mathrm{V})$ components is denoted here as $\Delta_{H V}$ and is calculated as:

$$
\Delta_{H V}(d B)=P L_{V H}(d)(d B)-P L_{V V}(d)(d B)
$$

This difference quantifies how much greater power the verticallyoriented (co-pol) antenna receives as compared to the horizontallyoriented antenna. For example, when the transmitting drone is in the South direction at $\mathrm{d}=20 \mathrm{~m}$ separation distance, $P L_{V V}=$ $88.16 \mathrm{~dB}$ and $P L_{V H}=93.84 \mathrm{~dB}$. As a result, $\Delta_{H V}$ is $5.68 \mathrm{~dB}$. This means that the vertically-oriented antenna can receive $5.68 \mathrm{~dB}$ higher RSS compared to the horizontally-oriented antenna at that location. In other words, despite matching the same polarization settings as the anechoic chamber, we record $7.5 \mathrm{~dB}$ less (Fig. 1(d) when mounted on a drone at $\phi=180^{\circ}$ ), which indicates that flight mechanics and/or relative drone headings can increase polarization mixing by more than $7 \mathrm{~dB}$ compared to the anechoic chamber measurements, where the drone body is fixed and does not move. Furthermore, we report that all values of $\Delta_{H V}$ here (in the four experiments) are within the NLOS condition of the XPD values reported in [35]. This might be due to the fact that the two drones do not exactly face each other in any of these four directions. The average $\Delta_{H V}$ value over all hovering locations when the transmitting drone is North is $2.24 \mathrm{~dB}$, while it is $8.55 \mathrm{~dB}$ and $5.34 \mathrm{~dB}$ when it is East and South, respectively. It is interesting to see that the lowest $\Delta_{H V}$ values were recorded for the North (which resulted previously in highest RSS). This means that when the transmitting drone is facing away, the transmitted polarization becomes almost independent of the received polarization, as the body of the transmitting drone completely changes the transmitted wave's polarization. These results indicate that higher average RSS values do not necessarily mean a higher decoupling of orthogonal polarizations (XPD) will be exhibited in air-to-air links that implement polarization diversity schemes as XPD becomes highly dependent (by more than $6 \mathrm{~dB}$ ) on the relative azimuth location of the transmitter.

To further give a better understanding of the statement made above, we compare the previous findings to a reference $\Delta_{H V}$, which we measure when the two drones are exactly facing each other and the antennas are mounted exactly at the same positions with the same separation distance of $20 \mathrm{~m}$. In this scenario, where no body blockage exists at neither the transmit nor the receiver drone, we find that $\Delta_{H V}=16 \mathrm{~dB}$, which is significantly greater than the values obtained from previous experiments in which the drone body resided along the path between the antennas. Also, this greater received power agrees with our results from the anechoic chamber 
measurements (Fig. 1(d)), signaling the loss is more due to the drone body than the in-flight vibrations. Furthermore, this value agrees with the LOS XPD values range found in [25,35]. With these experimental findings, we conclude that: $(i$.) the relative direction of a transmitter drone to another can affect both the average RSS in a co-polarized channel by $16 \mathrm{~dB}$ and the decoupling between orthogonally-polarized waves represented by XPD by more than $14 \mathrm{~dB}$, (ii.) unless the two drones are facing each other $\Delta_{H V}$, which represents XPD in our field experiments, is reported to fall in the NLOS range of the XPD values reported in [35], and (iii.) when the two drones are facing each other, we find that $\Delta_{H V}=16 \mathrm{~dB}$, which is the highest value compared to the different relative direction scenarios and agrees well with the LOS XPD measurements reported in $[25,35]$. These findings would be valuable to researchers when modeling and deploying UAV swarms that incorporate various antenna orientations and move freely in any direction.

Table 3: Additional Drone Body Losses Per Hovering Location When Tx Drone is in The North and South Directions

\begin{tabular}{|c|c|c|c|c|c|}
\hline Avg.loss & $20 \mathrm{~m}$ & $40 \mathrm{~m}$ & $60 \mathrm{~m}$ & $80 \mathrm{~m}$ & $100 \mathrm{~m}$ \\
\hline$\Gamma_{0^{\circ}}(d B)$ & 6.92 & 8.97 & 10.05 & 10.26 & 9.66 \\
\hline$\Gamma_{180^{\circ}}(d B)$ & 21.16 & 20.66 & 20.7 & 21.58 & 23.15 \\
\hline
\end{tabular}

\subsection{Body-Induced Effects on Predicting Large-Scale Fading}

In this section, we discuss how predicting the performance of UAVto-UAV links can give erroneous results if the blockage created by the different relative directions is not taken into consideration. We use the path-loss model given by $[12,14]$ :

$$
P_{r}=P_{t}-P_{L\left(d_{o}\right)}-10 \alpha \log \left(d / d_{o}\right)+\xi_{s}
$$

Here, $P_{r}$ and $P_{t}$ are the received and transmitted power (in $\mathrm{dBm}$ ), respectively. $P_{L}\left(d_{o}\right)$ is the path loss at a reference distance $\left(d_{o}\right)$ and is given by $P_{L}\left(d_{o}\right)=20 \log (4 \pi d o / \lambda)$, and $d$ is the distance between the transmitter and receiver. The shadowing parameter $\xi_{s}$ is normally distributed with zero mean, and a standard deviation $\sigma$ in $\mathrm{dB}$. Finally, $\alpha$ is the path loss exponent, which is known to depend largely on the surrounding environment of the transmitter and receiver and found to be close to 2 in many drone-to-drone links [7]. In this model, the transmit power is $6.2 \mathrm{dBm}$, and the standard deviation of the shadowing parameter is chosen to be $\sigma=2 \mathrm{~dB}$. The measured reference path loss $\left(P_{L}\left(d_{o}\right)\right.$ at $\left.d_{o}=20 \mathrm{~m}\right)$ with both UAVs facing each other $(\mathrm{PL}=67.7 \mathrm{~dB})$ is found to be very close to the free-space path-loss at this distance $(\mathrm{PL}=66.41 \mathrm{~dB})$, which indicates that when UAVs are facing each other. No additional drone body losses need to be included and the log-distance path-loss model can accurately describe such links [15].

However, this is not the case when drones move in different directions from each other. According to our measurements, and as we saw in Fig. 4, when the transmit drone moves in various relative directions with respect to the receiving drone, the body of the transmitting and receiving drones become an obstacle in the received signal's path causing significant reductions in the average RSS level (up to $16 \mathrm{~dB}$ ). We quantify the difference between the measured PL in the four directions and the PL predicted using the
Figure 5: Absolute error in prediction using our model and the conventional PL model.

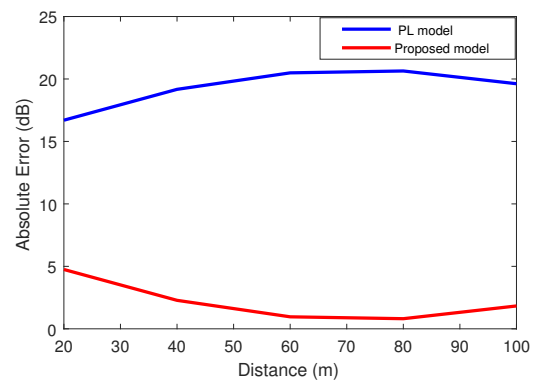

conventional model (which gave accurate predictions of the case when the two drones are facing each other) and propose a range of additional losses that should be added to the conventional model for improved prediction accuracy.

Based on the results of Fig. 4, we choose the North and South cases to cover the range of values of the additional loss that should be added to the model. This additional loss, denoted by $\Gamma_{\phi}$, is defined here as the difference between the average RSS of the case when both drones are facing each other (which is accurately predicted by the conventional PL model) and the average RSS when the transmit drone is at a certain $\phi$ direction. For example, in our case, the North and South directions of the Tx drone correspond to $\phi=0^{\circ}$ and $\phi=180^{\circ}$, respectively, with regards to the Rx drone location (see Fig. 3(a)). The values of $\Gamma_{\phi}$ at each hovering location for both directions are summarized in Table 3 . From the values given in the table, we can clearly see how the additional loss can be a function of the relative azimuth direction $(\phi)$ between the two drones. For example, when the Tx drone is North, the additional loss does not exceed $10.26 \mathrm{~dB}$. When it is South, however, it can reach up to $23 \mathrm{~dB}$. The minimum value of the additional loss is $\Gamma_{0^{\circ}}=6.92 \mathrm{~dB}$ and the maximum value is $\Gamma_{180^{\circ}}=23.15 \mathrm{~dB}$. Therefore, the modified path loss equation when the Tx drone takes different relative directions can be expressed as:

$$
P L_{U A V}=P L_{\text {log-distance }}+\Gamma_{\phi}
$$

Here, $P L_{\text {log-distance }}$ is the log-distance path loss model when the two drones are facing each other (no different relative directions). To demonstrate the applicability of this approach, the measured mean RSS values in the East direction are taken as an example. The measured values at the hovering locations can be found in Fig. 4. The predicted values (using the reference path loss when the drones are facing each other) are: $-60.5 \mathrm{dBm},-65.79 \mathrm{dBm},-69.85 \mathrm{dBm},-72.59$ $\mathrm{dBm}$, and $-74.02 \mathrm{dBm}$. On average, the absolute difference (absolute error) between the prediction made by the conventional model (that is very accurate and within $1 \mathrm{~dB}$ of accuracy when the two drones are facing each other) and the measured values when the Tx drone is East is $19.32 \mathrm{~dB}$. However, if we implement (4) using the average value of $\Gamma_{180^{\circ}}$, which is equal to $21.45 \mathrm{~dB}$, the absolute error is reduced to $2.12 d B$ (a 89\% decrease in error). The results are shown in Fig. 5. These findings may be generalized to other UAV-to-UAV scenarios where links are established at the same altitudes with negligible reflections from the ground [14] and the relative direction of one drone to the other is changing. 


\section{IMPACT OF ELEVATION ANGLE ON RSS AND DIVERSITY WITH DIFFERENT ANTENNA ORIENTATIONS}

It is well established in literature that the performance of MIMO systems highly depends on the spatial correlation of the channel matrix. This spatial correlation is found to vary according to changes in the channel induced by different antenna radiation patterns, spacing, orientation, polarization, and elevation and azimuth angle of arrivals [26, 27, 33, 34]. In addition, recent studies, such as [36], found that, in drone swarm applications, if all ground station antennas are identically oriented and a UAV is moving at different elevation angles, the received signal can be effectively lost due to polarization mismatch. This motivates us to experimentally investigate the effects of elevation angle on the RSS with various antenna orientations at the receiver drone in a $1 \times 2$ receive diversity system. First, we discuss the experiment procedure. Then, the effect of the elevation angle on RSS for different antenna orientations is analyzed. After that, we discuss antenna spacing and correlation and conclude the section with SNR gains due to diversity and their dependence on antenna orientation and elevation.

\subsection{Experiment Procedure}

In this set of experiments, the transmitting UAV is hovering at an altitude of $80 \mathrm{~m}$ with its transmitting antenna oriented vertically upward (VU), facing the receiving drone which moves around the transmitting UAV in a predefined sequence of hovering locations, creating a 3D shape (Fig. 6). Diversity is implemented at the receiving UAV which flies in an automated, repeatable fashion using waypoints and resulting in four distinct (negative) angles below the transmitter and four (positive) angles above the transmitter. The below and above points are separated by an elevation angle of $\theta_{\text {elev. }}=0^{\circ}$. The horizontal distance $\left(d_{h}\right)$ between the Tx and $\mathrm{Rx}$ drones is $20 \mathrm{~m}$ at $\theta_{\text {elev. }}=0^{\circ}$ and the angle-specific distance is $d_{\theta_{\text {elev }}}=\sqrt{d_{h}^{2}+d_{v}^{2}}$, where $d_{v}$ is the vertical distance, which varies from $-30 \mathrm{~m}$ (i.e., $50 \mathrm{~m}$ above ground) to $+30 \mathrm{~m}$ (i.e., $110 \mathrm{~m}$ above ground) in 10-m increments. The different elevation angles made (in sequence) are: $-90^{\circ},-56.3^{\circ},-45^{\circ},-26.5^{\circ}, 0^{\circ},+26.5^{\circ},+45^{\circ},+56.3^{\circ}$ and $+90^{\circ}$ and can be calculated as $\theta_{\text {elev }} .=\arctan \left(d_{v} / d_{h}\right)$.

Experiments are carried out for six different antenna orientation combinations (VU-VU, VU-VD, VU-H, VD-VD, H-H and H-VD) where VU, VD, and $\mathrm{H}$ represents vertical-up, vertical-down, and horizontal antenna orientations, respectively. See Fig. 6. The RSS is recorded at each hovering location for $30 \mathrm{~s}$ at a sampling rate of $32 \mathrm{k}$ samples/s and averaged over 10 seconds. The two UAVs are in a perfect LOS condition at a carrier frequency of $2.5 \mathrm{GHz} 5$ and a measured average noise floor of $-110 \mathrm{dBm}$. The average RSS values are fitted using a second-order polynomial in the range $\theta_{\text {elev. }}=$ $-56^{\circ}$ to $+56^{\circ}$, and the results are plotted and analyzed.

\subsection{Effect of Elevation Angle on RSS}

In this section, we study the dependence of RSS on the elevation angle between two drones for different antenna orientations. In general, if we look at Figs. 7(a) and 7(b), we observe an expected trend where the average RSS follows an arch-like shape in all verticallyoriented antennas in the range $\theta_{\text {elev }} .=-56^{\circ}$ to $+56^{\circ}$, with the

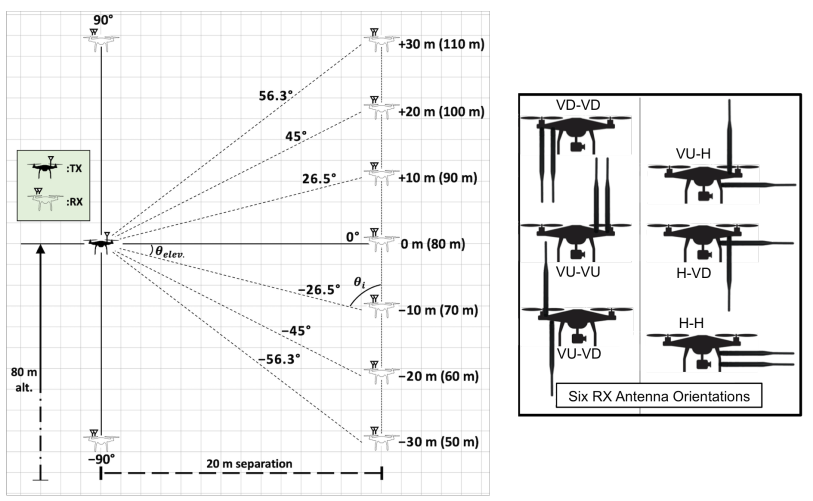

Figure 6: 3D experiment setup with $6 \mathrm{Rx}$ antenna orientation combinations in the $1 \times 2$ diversity system.

strongest average RSS recorded at an elevation angle of $\theta_{\text {elev. }}=0^{\circ}$; this is where the two drones exhibit perfect LOS at the same altitude. As the receiving drone starts moving up (to $110 \mathrm{~m}$ ) or down (to $50 \mathrm{~m}$ ), reductions in the signal level start to appear. These reductions are mainly caused by polarization mismatch and the elevation profile of the radiation pattern [18], which we characterized in the anechoic chamber (see Fig. 1(b)) for isolated (no drone, antenna only) and drone-mounted scenarios.

We first analyze results from the vertically-oriented receivers (VD-VD) (Fig. 7(b)) to understand the effect of elevation on RSS between two UAVs when the antennas used are identical with matched orientations (vertical). We can see that the two receiving antennas undergo the same behavior versus the elevation angle. The received signal level increases from around $-87 \mathrm{dBm}$ to -67 $\mathrm{dBm}(20 \mathrm{~dB}$ increase $)$ when the receiving drone moves from $-56.3^{\circ}$ to $0^{\circ}$ elevation angle. Then, as the drone moves higher (from $0^{\circ}$ to $+56^{\circ}$ elevation), the received signal level decreases from $-67 \mathrm{dBm}$ to $-85 \mathrm{dBm}$ (18 $\mathrm{dB}$ decrease), until it reaches around $-91 \mathrm{dBm}$ as it reaches exactly above the transmitting drone $\left(+90^{\circ}\right)$. When this receiving drone moves to the $-90^{\circ}$ elevation location (right below the Tx drone), an average RSS level of $-97 \mathrm{dBm}$ is reported. This trend is observed for all vertically-oriented receivers.

We conclude here that in an air-to-air links where two drones have the same antenna types and orientation, movement of the receiving drone at different elevation angles can reduce the signal level by up to $30 \mathrm{~dB}$. This $30 \mathrm{~dB}$ difference in RSS can be crucial when designing algorithms for optimal drone placement [19]. Similar findings in cellular to UAV and air-to-ground scenarios were reported in [18], and [10]. However, in addition to not covering air-to-air links, the proximity of the receiver or transmitter to the ground in both studies makes it difficult to isolate the elevation factor from multipath and the surrounding environment.

Furthermore, the nature of drone movement in 3D space and the low RSS levels measured by vertical antennas at $\theta_{\text {elev }}=|90|^{\circ}$ motivate us to employ polarization diversity [21] that is represented by using two co-located orthogonally-oriented antennas. If we look at Fig. 7(a), where we implement a horizontally-oriented $(\mathrm{H})$ receive antenna in addition to a vertically-oriented (VD) antenna, we can see that although VD results in higher RSS values throughout most elevation angles, around $+56^{\circ}$ the RSS for the $\mathrm{H}$ receiver starts to increase, where lower RSS values for the VD receiver are measured. 


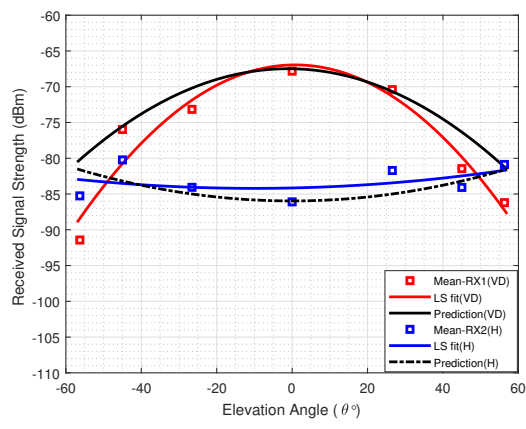

(a)

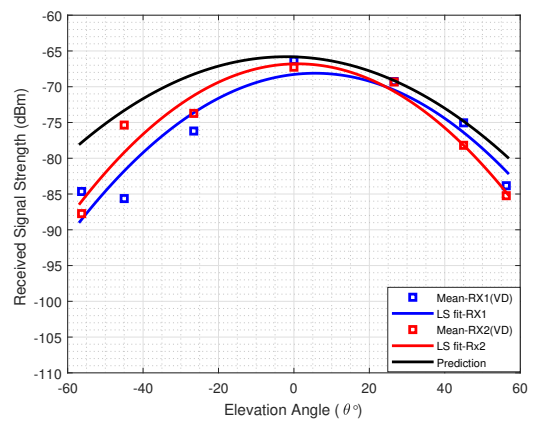

(b)

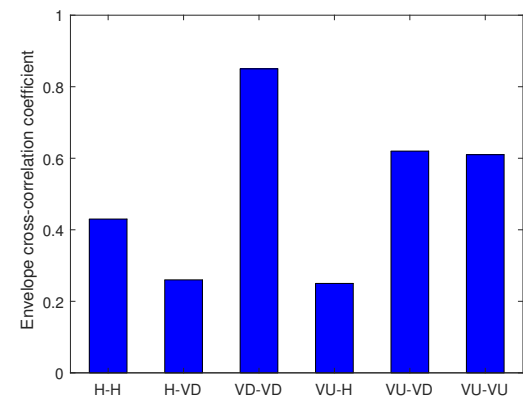

(c)

Figure 7: Average RSS vs. elevation angle for two different antenna orientation combinations: H-VD (a) and VD-VD (b). Correlation coefficient for the received signal envelope in the $1 \times 2$ drone-based system with different antenna orientations (c).

For example, at exactly $+90^{\circ}, \mathrm{H}$ is reported to measure an average RSS value of $-85.8 \mathrm{dBm}$, where VD results in an average RSS of $-98 \mathrm{dBm}$ (approx. $12 \mathrm{~dB}$ higher RSS at $\mathrm{H}$ ). In another example (VU$\mathrm{H}$ experiment) the $\mathrm{H}$ antenna captures $20 \mathrm{~dB}$ higher average RSS compared to the VU antenna. These results also agree with our anechoic chamber measurements (Fig. 1(d)) that show a measured received power of $16 \mathrm{~dB}$ higher for the $\mathrm{H}$ orientation.

To predict the average RSS and compare against the measured values, we add a polarization-mismatch loss [36] to the model in (3). Polarization-mismatch loss, also known as polarization loss factor (PLF), between a linearly-polarized (LP) incoming wave and $\mathrm{LP}$ antennas is a function of $\delta$, which is the difference between the antenna tilt angle $\left(\theta_{\text {tilt }}\right)$ and the incident angle of the incoming wave $\left(\theta_{i}\right)$ [17]. This PLF is given by $\operatorname{PLF}(d B)=20 \log (\cos (\delta))$. The reference received power is taken at $20 \mathrm{~m}$ when both drones are facing each other with vertical antennas. It is assumed that the location of the drone is known to the model; that is, for the same $d_{\theta_{\text {elev }}}$, it is known whether the drone is at a positive or a negative $\theta_{\text {elev. }}$. This is important because of the different direction-specific gain values of the antenna (see Fig. 1(b)). We include these radiation pattern gain values at the elevation angles as $G_{t}$ and $G_{r}$ for the transmit and receive antennas, respectively. A shadowing of $\sigma=1.5$ $\mathrm{dB}$ is used, and the prediction results are plotted in the same figures.

\subsection{Antenna Placement, Orientation and Correlation}

Since the orientation and spacing of two co-located receiving antennas can greatly affect the correlation and consequently the capacity of a MIMO system [29], we analyze the cross-correlation coefficient of the received signal at the two receiving branches in all of our six experiments. In doing so, we see how our antenna spacing decision of $\frac{2}{3} \lambda$ compares against what has been studied in literature and provide recommendations on antenna placement and polarization decisions. The correlation coefficient between the two received signal envelopes is calculated according to [34]:

$$
\rho_{i, j}=\frac{\sum_{n=1}^{N}\left(r_{i}-\overline{r_{i}}\right)\left(r_{j}-\overline{r_{j}}\right)}{\sqrt{\sum_{n=1}^{N}\left(r_{i}-\overline{r_{i}}\right)^{2}} \sqrt{\sum_{n=1}^{N}\left(r_{j}-\overline{r_{j}}\right)^{2}}}
$$

Here, $N$ is the total number of samples, and $\overline{r_{i}}$ is the mean value of the fast-fading signal envelope $r_{i}$, which corresponds to the first antenna orientation. The term $r_{j}$ corresponds to the second receiver's antenna orientation. For example, $\rho_{h, v d}$ is the correlation coefficient between the signal envelopes of the $\mathrm{H}$ and VD antennas in the H-VD experiment. We calculate this correlation coefficient for the signal envelopes received throughout the flight path mentioned above and find that, except for one antenna orientation combination (VD-VD), the correlation coefficient is found to always be less than 0.7. For example, the VU-VU and VU-VD experiments result in $\rho_{v u, v u}=0.61$ and $\rho_{v u, v d}=0.62$. Furthermore, the orthogonal antenna orientations ( $\mathrm{H}-\mathrm{VD}$ and $\mathrm{VU}-\mathrm{H})$ result in the lowest correlation coefficients (around 0.2) among all experiments, which can offer greater diversity gains. The obtained values from our $\frac{2}{3} \lambda$ antenna spacing and orientations are similar to the values found by [29] of $0.6 \lambda$ in which a correlation coefficient of 0.7 and 0.3 were found for the VU-VU and VU-H orientations, respectively. The correlation coefficient for the six antenna orientation combinations are shown in Fig. 7(c). Using these results and based on the objective (diversity or multiplexing gains), researchers can make informed decisions when selecting antenna orientation and spacing for drone communications.

\subsection{Elevation Impact on SNR Improvements for Different Antenna Orientations}

We now analyze the effect of elevation on the SNR improvements that can be achieved by selection diversity in all of the six experiments. The SNR improvement over a reference branch $i$ at an elevation angle $\theta$ is defined here (in $\mathrm{dB}$ ) as the expected value of the difference between the selected (maximum) SNR in the $1 \times 2$ setup over the reference branch. It is given by:

$$
\gamma_{i}^{\theta}=\mathbb{E}\left[S N R_{1 \times 2}^{\theta}-S N R_{i}^{\theta}\right]
$$

For example, in the VU-VD experiment, $\gamma_{1}$ and $\gamma_{2}$ indicate the SNR improvement over the VU and VD antenna orientations, respectively. Refer to Fig. 6 for the antenna orientations. As we saw earlier, different antenna polarizations can perform differently at different elevation angles due to the elevation radiation pattern and polarization mismatch. For example, the SNR improvement in the VU-H setup over $\mathrm{H}$ as the reference branch (i.e., $\gamma_{2}$ ) at $0^{\circ}$ elevation would be significantly different from the improvement 
at $45^{\circ}$ elevation. This is due to the fact that the highest SNR at $0^{\circ}$ angle was measured by the VU antenna, which matches the transmitter's antenna orientation and outperforms the SNR achieved by the horizontally-oriented antenna that receives a lower signal level. In contrast, at $|90|^{\circ}$ elevation, the $\mathrm{H}$ antenna, as mentioned above, records significantly higher SNR levels compared to the VU antenna, and the SNR improvement in this case $\left(\gamma_{1}\right)$ is significantly higher than $\left(\gamma_{2}\right)$. In Fig. 8, $\gamma_{H}$ and $\gamma_{V U}$ are the SNR improvements over the $\mathrm{H}$ and $\mathrm{VU}$ antenna orientations, respectively. We can see clearly that a higher SNR of $20 \mathrm{~dB}$ can be achieved around $0^{\circ}$ elevation due to the VU antenna orientation ( $\gamma_{H}$ is higher). Additionally, around $18.5 \mathrm{~dB}$ SNR improvement can be achieved around $+90^{\circ}$ elevation due to the $\mathrm{H}$ orientation ( $\gamma_{V U}$ is higher). These results agree with our expectations from the anechoic chamber that showed a $16 \mathrm{~dB}$ higher received power in $\mathrm{H}$ compared to $\mathrm{VU}$ at $90^{\circ}$ elevation. Furthermore, at $\theta_{\text {elev }} .=56^{\circ}, \gamma_{V U}$ becomes greater, indicating SNR improvements due to the $\mathrm{H}$ orientation over VU. This is due to the polarization mismatch mentioned in the previous sections. Namely, a vertically-oriented antenna $\left(\theta_{\text {tilt }}=0^{\circ}\right)$ at $\theta_{\text {elev. }}=56^{\circ}$ would exhibit a PLF of $-5.04 \mathrm{~dB}$. However, at the same angle, a horizontally-oriented antenna $\left(\theta_{\text {tilt }}=90^{\circ}\right)$ would exhibit a PLF of $-1.6 \mathrm{~dB}$, resulting in higher received power level. At $45^{\circ}$, the improvements are the lowest due to equal polarization mismatch between the two orientations, and improvements are strictly due to spatial diversity. Finally, to give an intuition for what these SNR improvements mean to the performance of 3D UAV-to-UAV links, let us take the $18.5 \mathrm{~dB}$ improvement at $\theta_{\text {elev }}=+90^{\circ}$ as an example. With an average measured noise floor of $-110 \mathrm{dBm}$, this improvement could enable a doubling ( $96 \%$ increase) in the spectral efficiency from $6.29 \mathrm{bps} / \mathrm{Hz}$ to $12.35 \mathrm{bps} / \mathrm{Hz}$, a significant improvement that could be crucial in applications that require high throughput.

The SNR improvement over the first and second branch in each experiment are summarized in Table 4 . We can clearly see that SNR improvements vary according to the different antenna orientations with the maximum not exceeding $10 \mathrm{~dB}$ in all co-polarized setups (first four columns). Also, we notice that in co-polarized setups, one antenna placement can dominate the other in terms of achieved SNR improvements. For example, in the VU-VU experiment, the improvement over the first branch is only $2.1 \mathrm{~dB}$ at one elevation angle. However, the improvement over the second branch can be up to 9.6 $\mathrm{dB}$. In the VU-VD setup, more than double the maximum improvement can be achieved due to the first branch $(8.7 \mathrm{~dB})$ compared to the second branch $(4 \mathrm{~dB})$. This shows how antenna placement decisions can affect SNR diversity improvements by more than $4 \mathrm{~dB}$. On average, cross-polarized receiving antennas achieve higher SNR gains than all co-polarized setups. Note that this is the case when the two drones are facing each other, and the average XPD value is $16 \mathrm{~dB}$. It is interesting to explore SNR diversity improvements when the two drones do not face each other. We expect for all improvements to be within the same range since all gains would be strictly due to spatial diversity because of low XPD values.

From these results, we conclude that having cross-polarized antennas when drones move in three dimensions is important, especially at angles above/below $45^{\circ}$ because of polarization mismatch losses. Measured improvements in SNR values of $20 \mathrm{~dB}$ can be achieved using cross-polarized receiving antennas, which can lead to an increase in throughput of $6 \mathrm{bps} / \mathrm{Hz}$. On the other hand, SNR
Figure 8: SNR improvement due to diversity in VU-H setup.

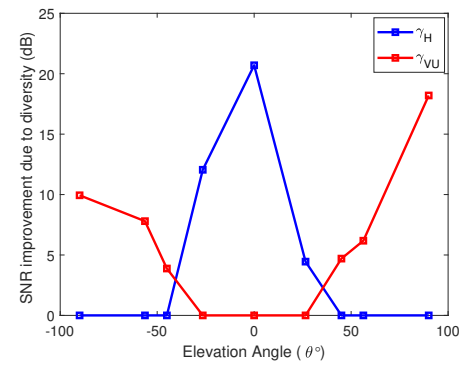

Table 4: SNR improvement (in $\mathrm{dB}$ ) due to diversity: improvement is with respect to branch $1\left(\gamma_{1}\right)$ and branch $2\left(\gamma_{2}\right)$

\begin{tabular}{|c|c|c|c|c|c|c|}
\hline$\gamma_{i}(\mathrm{~dB})$ & VU-VU & VD-VD & VU-VD & H-H & H-VD & VU-H \\
\hline$\gamma_{1}$ (max $)$ & 2.1 & 6.3 & 4.0 & 8.8 & 16.1 & 18.2 \\
\hline$\gamma_{1}($ avg $)$ & 2.1 & 1.1 & 1.7 & 4.3 & 4.5 & 5.6 \\
\hline$\gamma_{2}$ (max) & 9.6 & 8.5 & 8.7 & 0.3 & 11.5 & 20.7 \\
\hline$\gamma_{2}($ avg $)$ & 5.5 & 1.90 & 2.4 & 0.3 & 2.8 & 4.13 \\
\hline
\end{tabular}

improvements do not exceed $10 \mathrm{~dB}$ when using co-polarized receiving antennas, and the throughput increase does not exceed $2.5 \mathrm{bps} / \mathrm{Hz}$.

\section{RELATED WORK}

A few measurement-based studies have been recently conducted to understand drone-based communications. For example, an ultralow altitude drone to deliver $5 \mathrm{G}$ connectivity was implemented with different positions of a user on the ground [9]. Furthermore, in [7], air-to-ground experiments were performed with vertical and horizontal mobility of the transmit UAV with little emphasis on air-to-air links. In their work, the RSS was recorded at different elevations from the ground and for various rotations of the drone with two antenna orientation setups. Other work focused on the effect of having different antenna orientations with a fixed-wing UAV on IEEE 802.11a air-to-ground wireless link performance [8]. The work conducted by Niklas et al. helps in understanding the ground effects on multipath at a receiving UAV through measurements of an air-to-air link and the proposal of an empirical model that captures the variations of the Rician $K$ factor versus flight altitude [14]. Others focused on the network performance of three different network topologies and studies throughput versus distance [15]. Akram et al. modeled the cellular-to-UAV (CtU) channel through measurements conducted in a suburban environment [18]. The OpenAirInterface Software Alliance (OSA) deployed a UAVbased LTE relay that passes UDP packets to a moving target on the ground with a placement algorithm that is based on a predefined set of channel parameters [19]. Qualcomm reported results from experiments demonstrating connectivity and base station detection capability for a drone UE at different altitudes and carrier frequencies [20]. These studies, though relevant, do not investigate the impact of drone body on the radiation pattern in the elevation and azimuth spectrum and on XPD. Furthermore, none of the existing works experimentally characterize the elevation impact on AtA links with different antenna orientations and discuss antenna placement on drones. In this work, we cover these aspects in great detail 
and provided valuable insight that can help simulating, modeling, and deploying drone-based networks.

\section{CONCLUSION}

In this work, we presented results from experiments that mainly investigate the effects of the drone body and elevation on the performance of polarized UAV-to-UAV links. First, we analyzed the impact of relative direction and body obstruction on the RSS when two drones are in the same plane and showed that the average RSS can be reduced by up to $16 \mathrm{~dB}$ depending on the relative direction of the transmitter. Then, we investigated the impact of the drone body on polarization mixing and XPD and found that the body of the drone can manipulate the polarization of the incoming waves and reduce XPD by more than $20 \mathrm{~dB}$ compared to when the antenna is isolated and not mounted on a drone; a result that can be crucial when modeling polarized UAV-based MIMO channels. Second, we proposed a model that includes these additional body-induced losses for more accurate results when predicting the large-scale fading behavior of air-to-air links with different relative movements and directions in the azimuth plane. Third, we analyzed the impact of elevation angle on the RSS and the improvements due to diversity in a LOS UAV-to-UAV link for six different antenna orientation setups. In doing so, we found that the overall performance in three dimensions with various antenna orientations is mainly driven by the elevation angle. The RSS for the same antenna orientation can change dramatically (by up to $30 \mathrm{~dB}$ ) depending on the elevation angle of the receiving drone with respect to the transmitting drone. We also showed that a correlation coefficient less than 0.7 and reasonable diversity gains can be achieved with an antenna spacing of $\frac{2}{3} \lambda$ for both co-polarized and cross-polarized channels. We believe that these results can affect drone-based antenna placement and selection algorithms, optimization models that target drone placement, and future UAV-based channel models that currently fall short in capturing the discussed effects.

\section{ACKNOWLEDGEMENTS}

This work has been supported by the U.S. Department of Homeland Security (DHS), Countering Weapons of Mass Destruction (CWMD) Office, under a competitively awarded grant No. 18DNARI0002901-00. This support does not constitute an express or implied endorsement on the part of the Government. This work was also supported in part by the National Science Foundation under grants CNS-1526269 and CNS-1823304.

\section{REFERENCES}

[1] "Global Commercial Drones Market 2018-2022," in Business Wire, August 2018.

[2] S. Baidya, Z. Shaikh, and M. Levorato, "FlyNetSim: An Open Source Synchronized UAV Network Simulator based on ns-3 and Ardupilot," in Proc.of the 21st ACM Intern. Conference on Modeling, Analysis and Simulation of Wireless and Mobile Systems (ACM MSWiM), 2018.

[3] A. Al-Hourani, S. Kandeepan, and S. Lardner, "Optimal LAP Altitude For Maximum Coverage," in Proc. of IEEE Letters on Wireless Communications, December 2014.

[4] E. Kalantari, M. Shakir, H. Yanikomeroglu, and A. Yongacoglu, "Backhaul-aware Robust 3D Drone Placement in 5G+ Wireless Networks," in Proc. IEEE Intern. Conference on Communications Workshops (ICC Workshops), July, 2017

[5] R. Ghanavi, E. Kalantari, M. Sabbaghian, H. Yanikomeroglu, and A. Yongacoglu "Efficient 3D aerial base station placement considering users mobility by reinforcement learning," in Proc. of IEEE WCNC, 2018.

[6] M. Mozaffari, W. Saad, M. Bennis, and M. Debbah, "Mobile Internet of Things: Can UAVs Provide an Energy-Efficient Mobile Architecture?," Proc. of IEEE Global
Communications Conference (GLOBECOM), 2016.

[7] E. Yanmaz, R. Kuschnig, and C. Bettstetter, "Achieving air-ground communications in 802.11 networks with three-dimensional aerial mobility," in Proc. of IEEE Intern. Conf. Comp. Commun. (INFOCOM), Mini conference,, April 2013.

[8] C.-M. Cheng, P.-H. Hsiao, H. Kung, and D. Vlah, "Performance measurement of 802.11a wireless links from UAV to ground nodes with various antenna orientations," in Proc. of Intl. Conf. Computer Comm. and Networks, October 2006.

[9] Philip A. Catherwood, Brendan Black, Ebrahim Bedeer, Adnan Ahmad Cheema, Joseph Rafferty, James A. D. McLaughlin,"Radio Channel Characterization of MidBand 5G Service Delivery for Ultra-Low Altitude Aerial Base Stations," IEEE Access 7: 8283-8299, Jan. 2019.

[10] E. Yanmaz, R. Kuschnig, and C. Bettstetter, "Channel measurements over 802.11abased UAV-to-ground links," in Proc. Intern. Workshop on Wireless Networking for Unmanned Autonomous Vehicles, 2011.

[11] T. Willink, C. Squires, G. Colman, and M. Muccio, "Measurement and characterization of low altitude air-to-ground MIMO channels," IEEE Trans. Veh. Technol., vol. 65, no. 4, Apr. 2016

[12] Y. Shi, R. Enami, J. Wensowitch, and J. Camp, "UABeam: UAV-Based Beamforming System Analysis with In-Field Air-to-Ground Channels," Proc. of 15th Annual IEEE Intern. Conference on Sensing, Communication, and Networking (SECON), 2018.

[13] J. Chen, B. Daneshrad, and W. Zhu, "MIMO performance evaluation for airborne wireless communication systems," in Proc. IEEE MILCOM, 2011.

[14] N. Goddemeier and C. Wietfeld, "Investigation of Air-to-Air Channel Characteristics and a UAV Specific Extension to the Rice Model," Proc. of IEEE Globecom Workshops (GC Wkshps), 2015.

[15] E. Yanmaz, S. Hayat, J. Scherer, and C. Bettstetter, "Experimental Performance Analysis of Two-Hop Aerial 802.11 Networks," in Proc. of IEEE WCNC, 2014.

[16] D. Tse and P. Viswanath, "Fundamentals of Wireless Communication," Cambridge University Press, 2005.

[17] W. Stutzman and G. Thiele, "Antenna Theory and Design," 3rd Ed. Wiley, 2013.

[18] A. Al-Hourani and K. Gomez, "Modeling Cellular-to-UAV Path-Loss for Suburban Environments," in IEEE Wireless Communications Letters, vol. 7, no.1, Feb. 2018.

[19] R. Gangula, O. Esrafilian, D. Gesbert, C. Roux, F. Kaltenberger, and R. Knopp, "Flying Rebots: First Results on an Autonomous UAV-Based LTE Relay Using Open Airinterface," Proc. of IEEE 19th International Workshop on Signal Processing Advances in Wireless Communications (SPAWC), June 2018.

[20] "LTE Unmanned Aircraft Systems: Trial Report," Qualcomm, May 2017.

[21] R. Vaughan, "Polarization Diversity in Mobile Communications," IEEE Transactions on Vehicular Technology, Vol. 39, No. 3, August 1990, pp. 177-186

[22] R. G. Vaughan and J. B. Andersen, "Antenna diversity in mobile communications," IEEE Trans. Veh. Technol., vol. VT-36, Nov. 1987.

[23] V. Rodriguez, "Basic Rules for Indoor Anechoic Chamber Design [Measurements Corner]," IEEE Antennas Propagat. Mag., Vol. 58, No. 6, Dec. 2016

[24] S.-C. Kwon and G. L. St Âluber, "Geometrical theory of channel depolarization," IEEE Trans. Veh. Technol., vol. 60, Oct. 2011

[25] M. Shafi, M. Zhang, A. Moustakas, P. Smith, A. Molisch, F. Tufvesson, and S. Simon, "Polarized MIMO channels in 3-D: models, measurements and mutual information," IEEE J. Select. Areas Commun., vol. 24, no. 3, Mar. 2006

[26] "Spatial channel model for multiple input multiple output MIMO simulations," 3GPP, vol. TR 25.996, v6.1.0, Sep. 2003

[27] J. Wang, J. Zhao, and X. Gao, "Modeling and analysis of polarized MIMO channels in 3D propagation environment," in PIMRC, 2010.

[28] X. Su, D. Choi, X. Liu, and B Peng, "Channel Model for Polarized MIMO Systems With Power Radiation Pattern Concern," IEEE Access, vol. 4, Mar. 2016

[29] M.-T. Dao, V.-A. Nguyen, Y.-T. Im, S.-O. Park, and G. Yoon, "3D polarized channel modeling and performance comparison of MIMO antenna configurations with different polarizations," IEEE Trans. Antennas Propag., vol. 59, no. 7, 2011.

[30] L. Zeng, X. Cheng, C.-X. Wang, and X. Yin, "A 3D geometry-based stochastic channel model for UAV-MIMO channels," in Proc. IEEE WCNC, Mar. 2017.

[31] H. Jiang, Z. Zhang, L. Wu, and J. Dang, "Three-dimensional geometry based UAVMIMO channel modeling for A2G communication environments," IEEE Commun. Lett., vol. 22, no. 7, Jul. 2018.

[32] Y. Xing, O. Kanhere, S. Ju, T. S. Rappaport, and G. R. MacCartney Jr., "Verification and calibration of antenna cross-polarization discrimination and penetration loss for millimeter wave communications," 2018 IEEE 88th Vehicular Technology Conference (VTC2018-Fall), Chicago, USA, Aug. 2018.

[33] Y.-G. Lim, Y. J. Cho, T. Oh, Y. Lee, and C.-B. Chae, "Relationship between crosspolarization discrimination (XPD) and spatial correlation in indoor small-cell MIMO systems," IEEE Wireless Commun. Lett., vol. 7, no. 4, Aug. 2018

[34] Y. Yao, J. Zheng, and Z. Feng,"Diversity measurements for on-body channels using a tri-polarization antenna at $2.45 \mathrm{GHz}$, IEEE Antennas Wirel. Propag. Lett., 2012.

[35] T. Neubauer and P. C. F. Eggers, "Simultaneous characterization of polarization matrix components in pico cells," in Proc. Veh. Technol. Conf., 1999.

[36] P. Chandhar, D. Danev, and E. G. Larsson, "Massive MIMO for communications with drone swarms," IEEE Transactions on Wireless Communications, vol. 17, no. 3, March 2018. 